\title{
Prevalência de Parasitos Intestinais e Aspectos Socioambientais em Comunidade Indígena no Distrito de lauaretê, Município de São Gabriel da Cachoeira (AM), Brasil'
}

\section{Prevalence of Intestinal Parasites and Social-Environmental Aspects in an Indigenous Community in the lauaretê District, Municipality of São Gabriel da Cachoeira (State of Amazonas), Brazil'}

\author{
Leonardo Rios \\ Uniara - Centro Universitário de Araraquara. Prof. Titular da Es- \\ cola de Engenharia de Piracicaba. \\ Endereço: Av. Miguel Damha, 1400, Residencial Damha II, casa 323, \\ Jardim Guanabara, cep 13565-814, São Carlos, São Paulo, Brasil. \\ E-mail: leoriosळterra.com.br.

\section{Silvana Audrá Cutolo} \\ Faculdade de Saúde Pública/USP. E-mail: cutolosaœusp.br \\ Leandro Luiz Giatti \\ CPqL\&MD - Fiocruz /Amazônia e UEA - Universidade do Estado do \\ Amazonas
}

\section{Mário de Castro}

Instituto de Ciências Matemáticas e de Computação-ICMC/USP

\section{Aristides Almeida Rocha}

Faculdade de Saúde Pública/USP. E-mail: arirocha®usp.br

\section{Renata Ferraz de Toledo}

INPA - Instituto Nacional de Pesquisas da Amazônia

Maria Cecília Focesi Pelicioni

Faculdade de Saúde Pública/USP

Luciana Pranzetti Barreira

Faculdade de Tecnologia Flamingo

\section{Jéferson Gaspar dos Santos}

Centro de Patologia Clínica Campana

I Esta pesquisa foi financiada pela Fundação Nacional de Saúde (Funasa), por meio do convênio 513/04 com a Universidade de São Paulo. Parecer favorável no Conselho Nacional de Ética em Pesquisa (registro 10848) e ingresso na Terra Indígena autorizado pela Fundação Nacional do Índio (processo 0480/04). 0 estudo de registros de dados epidemiológicos provenientes de inquérito parasitológico recebeu parecer favorável do Comitê de Ética em Pesquisa da Universidade Federal do Amazonas (registro 157/2005).

Renata Ferraz de Toledo e Leandro Luiz Giatti são bolsistas de desenvolvimento científico regional - CNPq/FAPEAM.

\section{Resumo}

Situado em Terra Indígena do Alto Rio Negro, Iauaretê é o segundo maior pólo de concentração humana no município de São Gabriel da Cachoeira (AM) e agrega dez vilas, com 15 etnias. Esse distrito indígena vem sofrendo processo de transformação progressiva de padrão de ocupação disperso e ribeirinho para núcleo com feições urbanas, com aproximadamente 2700 habitantes, desencadeando problemas de saúde pública. Com objetivo de investigar a distribuição de prevalências de parasitos intestinais na população local e discutir a relação desse indicador de saúde com aspectos socioambientais no distrito de Iauaretê, foram realizadas análises da qualidade da água de abastecimento e exames parasitológicos em amostras de fezes e solo. Do total de amostras de água analisadas, $89,2 \%$ apresentaram resultado positivo para coliformes termotolerantes. Identificou-se que a vila Dom Bosco foi a que apresentou maior índice de prevalência em amostras fecais com $76 \%$ dos indivíduos parasitados. A vila São José apresentou o menor índice com $56 \%$. Os parasitos mais prevalentes em amostras fecais positivas foram, em ordem decrescente, Ascaris lumbricoides (64,84\%), Entamoeba coli (32,58\%), Endolimax nana (14,84\%) e Blastoyistis hominis (13,39\%). Em amostras de solo as prevalências mais significativas foram de ovos de Ascaris sp (52,6\%), cistos de Entamoeba coli $(52,6 \%)$ e larvas de ancilostomídeos (52,6\%). Concluiuse que os altos índices de parasitoses intestinais na população, aliados à falta de saneamento básico e à manutenção de práticas sanitárias tradicionais constituem um quadro preocupante em saúde pública. 
Palavras-chave: Saúde indígena; Saúde ambiental; Saúde pública; Parasitos intestinais.

\section{Abstract}

The Iauaretê District is the second largest human concentration centre in the municipality of São Gabriel da Cachoeira, state of Amazonas. Situated in Alto Rio Negro Indigenous Land, on the border between Brazil and Colombia, Iauaretê aggregates ten villages, encompassing 15 ethnic cultures. This indigenous district has been undergoing a process of progressive transformation from a disperse and riparian occupation pattern into a settlement with urban features, with approximately 2,700 inhabitants, triggering public health problems. Aiming to investigate the distribution of the prevalence of intestinal parasites in the local population and to discuss the relation between this health indicator and socio-environmental aspects in the Iauaretê district, the quality of the drinking water was analyzed and parasitological exams of excreta and soil samples were conducted. Of the total of analyzed water samples, $89.2 \%$ showed positive result for thermotolerant coliforms. The Dom Bosco village was the one presenting the highest prevalence index in excreta samples, with $76 \%$ of the individuals infested. The São José village presented the lowest index (56\%). The most prevalent parasites were, in decreasing order: Ascaris lumbricoides (64.84\%), Entamoeba coli (32.58\%), Endolimax nana (14.84\%), and Blastoyistis hominis (13.39\%). The most significant prevalences of parasites in soil samples were Ascaris sp eggs (52.6\%), Entamoeba coli cysts (52.6\%) and hookworms larvae (52.6\%). In conclusion, high indexes of intestinal parasitism in the population, together with lack of basic sanitation and maintenance of traditional sanitary practices are matters of concern in the public health area.

Keywords: Indigenous Health; Environmental Health; Public Health; Intestinal Parasites.

\section{Introdução}

Condições socioambientais se fazem relevantes em estudo de perfil epidemiológico de grupos e subgrupos sociais específicos, sobretudo em se tratando de populações indígenas que ocupam ambientes em condições sanitárias inapropriadas e que, ocasionalmente, concentram-se em grupos populacionais de pluralidade étnica.

Segundo Santos e Coimbra Jr. (2003), os povos indígenas no Brasil possuem um complexo e dinâmico quadro de saúde, diretamente relacionado a processos históricos de mudanças sociais, econômicas e ambientais, aliadas à expansão de frentes demográficas e econômicas da sociedade nacional nas diversas regiões do país. Atualmente, verifica-se a emergência de desafios à saúde dos povos indígenas, em conseqüência de doenças crônicas não-transmissíveis, doenças transmissíveis e contaminação ambiental, entre outros. Esses autores ressaltam que o perfil epidemiológico dos povos indígenas é muito pouco conhecido, o que decorre da exigüidade de investigações, da ausência de inquéritos e censos, bem como da precariedade de informações sobre morbidade e mortalidade.

As populações indígenas sempre foram negligenciadas pelas autoridades, e a assistência à saúde dos grupos indígenas sempre foi desorganizada e esporádica (Costa, 1987). Buchillet (1995) ressalta que para atender as demandas, as expectativas e as necessidades em matéria de saúde de uma comunidade indígena há uma necessidade de conhecimento acurado do perfil epidemiológico e sanitário. Isso é relevante em região que constitui o hábitat de vários povos, com distintos modos de uso e adaptação ao meio ambiente.

Este artigo objetiva investigar a distribuição de prevalências de parasitos intestinais em população indígena e discutir a relação desse indicador de saúde com aspectos socioambientais na sede do distrito de Iauaretê.

\section{Material e Métodos}

A sede do distrito de Iauaretê, no município de São Gabriel da Cachoeira, Estado do Amazonas (AM), constitui caso único em termos de concentração populacional em interior de Terra Indígena, onde ocorre significativo processo de transformação progressiva de padrão de ocupação de disperso e ribeirinho para nú- 
cleo com feições urbanas, composto de 10 vilas agregadas, onde ocorrem 15 etnias, concentrando aproximadamente 2700 habitantes (Andrello, 2004), modificando significativamente o ambiente e impondo necessidade de adaptação da população a novos hábitos de vida. Sendo o segundo maior pólo de concentração humana no município de São Gabriel da Cachoeira, a sede do distrito de Iauaretê situa-se na Terra Indígena do Alto Rio Negro, área fronteiriça entre Brasil e Colômbia, nos arredores da foz do Rio Papuri, no médio Rio Waupés, principal tributário do Alto Rio Negro.

No estudo, dados de número de habitantes e número de domicílios, sexo, idade e etnia da população foram obtidos por meio da análise de documentos no Distrito Sanitário Especial Indígena (DSEI) do Alto Rio Negro.

A qualidade de água utilizada para o abastecimento das populações locais foi estudada. Para isso, efetuou-se análise microbiológica em 35 amostras coletadas no mês de maio de 2005 e em 30 amostras coletadas no mês de julho do mesmo ano, de acordo com as fontes de captação de água disponíveis em cada um desses momentos nas distintas vilas da sede do distrito. Foi utilizada a metodologia Colilert (APHA, 1999), em amostras de 100 mL coletadas em saco plástico estéril, contendo EDTA, ao qual era adicionado substrato cromogênico ONPG-MUG. O material era incubado em estufa microbiológica a $36^{\circ} \mathrm{C}$ durante 24 horas. Posteriormente procedia-se leitura com lâmpada ultravioleta para o diagnóstico da presença ou da ausência de coliformes totais e Escherichia coli (coliformes termotolerantes), indicadores de contaminação por material fecal.

Foi realizado um inquérito coproparasitológico no mês de maio de 2005 por parte do DSEI do Alto Rio Negro, com apoio da Faculdade de Saúde Pública da Universidade de São Paulo e do Centro de Pesquisa Leônidas e Maria Deane - Fiocruz/Amazônia, para verificar a prevalência de parasitos intestinais na comunidade. Considerou-se para o cálculo desse indicador tanto os helmintos e protozoários parasitos quanto os comensais, tais como $\varepsilon$. coli, $\varepsilon$. nana e I. butschlii. Um cálculo amostral tendo como referência a população local de 2700 habitantes e uma margem de erro de $5 \%$, indicou a necessidade de se efetuar 336 amostras para exames parasitológicos fecais. Com efeito, há de se considerar algumas dificuldades no sentido de aplicar metodologia de inquérito parasitológico na área de estudo. Mesclam-se localmente dificuldades logísticas, culturais e de mobilidade populacional, incidindo em problemas na distribuição de amostras, localização de moradores e sua adesão ao inquérito. Nesse sentido, optou-se pela distribuição de frascos para coleta em número superior ao $\mathrm{N}$ amostral acima indicado.

Inicialmente foram distribuídos 1450 frascos de Coprotest pelos agentes indígenas de saúde, em duas etapas, de maneira proporcional ao número de habitantes de cada vila. Foi necessário contar com adesão da população para a realização dos exames, algo que permitiu a distribuição das amostras, que eram então recebidas e identificadas com a vila, o nome, sexo, idade e etnia, numeradas e relacionadas em uma tabela. Desse modo, foram realizados exames parasitológicos em 895 indivíduos.

Para a obtenção do indicador de saúde intestinal que é o exame parasitológico fecal e diante das dificuldades logísticas foi decidido aplicar apenas uma técnica de análise. Desse modo, todas as amostras recebidas foram encaminhadas a laboratório e, então, submetidas ao método de sedimentação espontânea em tubo cônico de $12 \mathrm{~mL}$, para posterior análise em microscópio óptico comum, segundo Lutz (1919).

Também foram executadas análises parasitológicas de amostras de solo no mês de julho de 2005 . Procedeu-se assim, amostragem em área equivalente a 158,5 hectares, delimitada de acordo com a distribuição dos domicílios na sede do distrito de Iauaretê. Foram coletadas 225 amostras de solo. A distribuição espacial das amostras foi realizada pela técnica de quarteamento da ABNT - NBR 10007 (1987), tendo por referência a distribuição espacial dos domicílios. Com o auxílio de pá, foram coletadas cerca de 300 gramas de solo por ponto de amostragem e acondicionadas em sacos estéreis com especificações dos pontos por vila. Para análise, utilizou-se 5 gramas de solo por ponto de amostragem. As amostras foram homogeneizadas com água de diluição e detergente Tween 8o; em seguida, filtradas para remoção de materiais grosseiros; e centrifugadas a $1800 \mathrm{rpm}$ por cinco minutos; o líquido sobrenadante foi retirado e o sedimento foi ressuspendido com solução de sulfato de zinco a 33\% (densidade 1.2). Foram identificados cistos de protozoários, larvas e ovos de helmintos de acordo com referências e Atlas de Parasitologia Médica 
(Cutolo e col., 2006; Leventhal e Cheadle, 200o; OMS, 1992; Peters e Gilles, 1995; Whitfield, 1993).

Para as análises estatísticas foi utilizado o sistema estatístico R (R Development Core Team, 2005). As técnicas utilizadas compreendem gráficos, estatísticas descritivas, teste qui-quadrado de proporções, teste de Wilcoxon, teste de Kruskal-Wallis e o coeficiente de correlação de Spearman com nível de significância de $5 \%$.

\section{Resultados}

As 10 vilas da sede do distrito se distribuem em sete na margem esquerda do Rio Waupés e três na margem direita (Figura 1). Número de habitantes, de domicílios e ocorrência de etnias encontram-se distribuídos por vila apresentados na Tabela 1.
Figura I - Distribuição das vilas da sede do distrito de lauaretê, município de São Gabriel da Cachoeira / AM

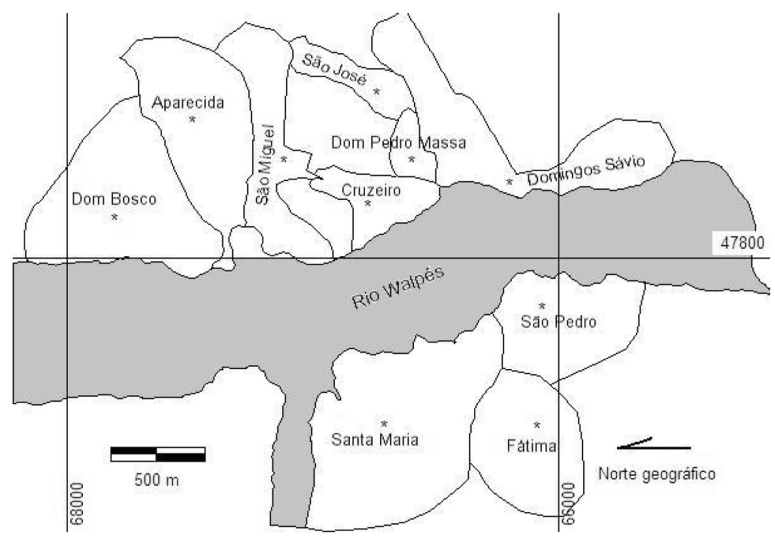

Tabela I - Etnias, número de habitantes, amostragens, número de domicílios e prevalências de parasitos intestinais e de coliformes termotolerantes por vila na sede do Distrito de lauaretê, município de São Gabriel da Cachoeira / AM, 2005-2006

\begin{tabular}{|c|c|c|c|c|c|c|c|c|c|c|}
\hline Vila & $\begin{array}{l}\text { Etnias mais } \\
\text { representativas }\end{array}$ & $\begin{array}{l}\mathrm{N} \\
\text { habit }\end{array}$ & $\begin{array}{l}\mathrm{N} \\
\text { amostras } \\
\text { de fezes }\end{array}$ & $\begin{array}{l}\mathrm{N} \\
\text { domicílios }\end{array}$ & $\begin{array}{l}\mathrm{N} \\
\text { amostras } \\
\text { de solo } \\
\text { coletadas }\end{array}$ & $\begin{array}{l}\text { \% de } \\
\text { prevalência } \\
\text { de parasitos } \\
\text { intestinais }\end{array}$ & $\begin{array}{l}\text { \% de } \\
\text { prevalência } \\
\text { para mais } \\
\text { de um } \\
\text { parasito } \\
\text { intestinal }\end{array}$ & $\begin{array}{l}\text { \% de } \\
\text { prevalência } \\
\text { para Ascaris } \\
\text { dentre } \\
\text { amostras } \\
\text { fecais } \\
\text { positivas }\end{array}$ & $\begin{array}{l}\text { \% de } \\
\text { prevalência } \\
\text { de ovos de } \\
\text { Ascaris } \\
\text { no solo, } \\
\text { dentre as } \\
\text { positivas }\end{array}$ & $\begin{array}{l}\% \text { de } \\
\text { amostras } \\
\text { de água } \\
\text { com } \\
\text { coliformes } \\
\text { termoto- } \\
\text { lerantes }\end{array}$ \\
\hline Aparecida & $\begin{array}{l}\text { Tariano, Tukano, } \\
\text { Piratapuia }\end{array}$ & 425 & 103 & 70 & 34 & 73 & 33 & 53,40 & 23,52 & 100 \\
\hline Cruzeiro & $\begin{array}{l}\text { Tariano, Tukano, } \\
\text { Piratapuia }\end{array}$ & 376 & 105 & 61 & 31 & 59 & 27 & 38,10 & 0,00 & 70 \\
\hline D. Bosco & $\begin{array}{l}\text { Tariano, Tukano, } \\
\text { Wanano }\end{array}$ & 332 & 87 & 56 & 28 & 76 & 39 & 58,62 & 24,14 & 100 \\
\hline $\begin{array}{l}\text { D. Pedro } \\
\text { Massa }\end{array}$ & $\begin{array}{l}\text { Tariano, Tukano, } \\
\text { Piratapuia }\end{array}$ & 276 & 117 & 47 & 24 & 74 & 38 & 56,03 & 8,33 & 100 \\
\hline $\begin{array}{l}\text { Dom. } \\
\text { Sávio }\end{array}$ & $\begin{array}{l}\text { Tukano, Tariano, } \\
\text { Piratapuia }\end{array}$ & 215 & 65 & 40 & 20 & 75 & 42 & 43,08 & 5,00 & 50 \\
\hline Fátima & Hupda* & 120 & 33 & 15 & 10 & 67 & 21 & 45,45 & 10,00 & 100 \\
\hline S. Maria & $\begin{array}{l}\text { Tariano, Tukano, } \\
\text { Piratapuia }\end{array}$ & 290 & 106 & 46 & 23 & 73 & 37 & 36,79 & 4,34 & 100 \\
\hline S. José & $\begin{array}{l}\text { Piratapuia, } \\
\text { Tukano, Desana }\end{array}$ & 211 & 105 & 34 & 17 & 56 & 24 & 38,68 & 23,53 & 100 \\
\hline S. Miguel & $\begin{array}{l}\text { Tariano, Tukano, } \\
\text { Piratapuia }\end{array}$ & 343 & 121 & 54 & 20 & 76 & 40 & 45,55 & 35,00 & 100 \\
\hline S. Pedro & $\begin{array}{l}\text { Tariano, } \\
\text { Piratapuia, Juriti }\end{array}$ & 118 & 52 & 17 & 15 & 67 & 27 & 25,00 & 13,33 & 75 \\
\hline
\end{tabular}

* Em Vila Fátima apenas 4 pessoas não são declaradas como de etnia Hupda 
Tabela 2 - Características demográficas da população amostrada na sede do distrito de lauaretê (AM), 2005-2006

\begin{tabular}{|c|c|c|c|}
\hline \multirow[t]{2}{*}{ Sexo } & Feminino & 471 & 52,80 \\
\hline & Masculino & 421 & 47,20 \\
\hline Idade & $01-10$ & 309 & 34,68 \\
\hline \multirow[t]{7}{*}{ (anos) } & $101-20$ & 188 & 21,10 \\
\hline & $20+30$ & 83 & 9,32 \\
\hline & $30+40$ & 74 & 8,31 \\
\hline & $40 \vdash 50$ & 69 & 7,74 \\
\hline & $50+60$ & 92 & 10,32 \\
\hline & $601-80$ & 66 & 7,41 \\
\hline & $80+120$ & 10 & 1,12 \\
\hline \multirow[t]{14}{*}{ Etnia } & Tariano & 311 & 35,26 \\
\hline & Tukano & 237 & 26,87 \\
\hline & Piratapuia & 130 & 14,74 \\
\hline & Dessano & 67 & 7,60 \\
\hline & Wanano & 37 & 4,20 \\
\hline & Hupda & 33 & 3,74 \\
\hline & Arapaço & 30 & 3,40 \\
\hline & Caboclo & 12 & 1,36 \\
\hline & Baré & 9 & 1,02 \\
\hline & Juriti & 7 & 0,79 \\
\hline & Cubeo & 3 & 0,34 \\
\hline & Mestiço & 3 & 0,34 \\
\hline & Baniwa & 2 & 0,23 \\
\hline & Não Índio & 1 & 0,11 \\
\hline
\end{tabular}

Distribuições da população amostrada por gênero, etnia e faixas etárias são apresentadas na Tabela 2.

Do total de amostras de água analisadas, 89,2\% apresentaram contaminação por coliformes termotolerantes. Na Tabela 1, apresentam-se os resultados de percentual de amostras positivas para coliformes termotolerantes por vila, de acordo com as fontes de água disponíveis em período de coleta. Identificou-se que as vilas Dom Bosco, Aparecida, São Miguel, Fátima, Santa Maria, Dom Pedro Massa e São José mostraram os maiores índices de contaminação por coliformes termotolerantes em fontes de água disponíveis (100\% das amostras). Domingos Sávio foi a vila que apresentou o menor índice (50\%).

Os exames parasitológicos de fezes realizados mostraram as maiores prevalências para as vilas Dom Bosco, São Miguel e Domingos Sávio (Tabela 1), com índices de indivíduos parasitados variando entre $75 \%$ e $76 \%$. As vilas Cruzeiro e São José foram as que apresentaram as menores índices, ficando entre $56 \%$ a 59\% dos indivíduos parasitados.

0 parasito encontrado isoladamente com a maior prevalência foi o Ascaris lumbricoides, que também está entre o grupo de parasitos que mais apareceu nos resultados com mais de uma espécie. Dom Bosco e Dom Pedro Massa foram as vilas que apresentaram as menores prevalências para esse helminto (41\% e 43\%, respectivamente) entre as amostras fecais positivas. Já a vila São Pedro foi a que apresentou a maior porcentagem de indivíduos com esse parasito dentre os resultados positivos para parasitos intestinais (75\%).

Indivíduos albergando mais de uma espécie de parasito (poliparasitismo ou infecções múltiplas) também apresentaram um alto índice de prevalência dentre a população amostrada. As vilas Domingos Sávio (42\%) e São Miguel (40\%) foram as que apresentaram os maiores valores de poliparasitados, já as vilas São José e Fátima apresentaram os menores valores, respectivamente $24 \%$ e $21 \%$ (Tabela 1 ).

Na Tabela 2, estão apresentadas algumas características da amostra populacional para exames de fezes, com a distribuição por vila, sexo, idade e etnia. As vilas São Miguel, Domingos Sávio, Santa Maria e São José concentraram 44,4\% dos sujeitos (Tabela 1). Predominaram sujeitos do sexo feminino, mas a diferença em relação à proporção de sujeitos do sexo masculino não foi significativa. A idade dos sujeitos variou de o,75 a 113 anos, com média, mediana e desvio padrão iguais a 25,1; 15,9 e 21,7 anos, respectivamente. A amplitude e o desvio-padrão das idades indicaram grande variabilidade na distribuição etária. Menores de 10 anos constituíram o grupo etário mais expressivo $(34,68 \%)$ e cerca de $90 \%$ dos sujeitos tinham idade inferior a 60 anos. Sujeitos da etnia Tariano constituíram a maioria $(35,26 \%)$ e um pouco mais de $3 / 4$ dos sujeitos $(76,87 \%)$ eram das etnias Tariano, Tukano e Piratapuia. Conforme a Figura 2, as mulheres apresentaram prevalência de parasitoses variando entre $46 \%$ e $81 \%$ e os homens, entre $58 \%$ e $80 \%$. A vila Fátima respondeu com menor número de participantes (Tabela 1).

De acordo com a Figura 3, verificou-se a presença de uma única espécie de parasito (monoparasitismo) em $36 \%(322 / 895)$ das amostras de fezes e polipara- 
Figura 2 - Prevalência de sujeitos com parasitose de acordo com a vila de procedência e o sexo, 2005-2006

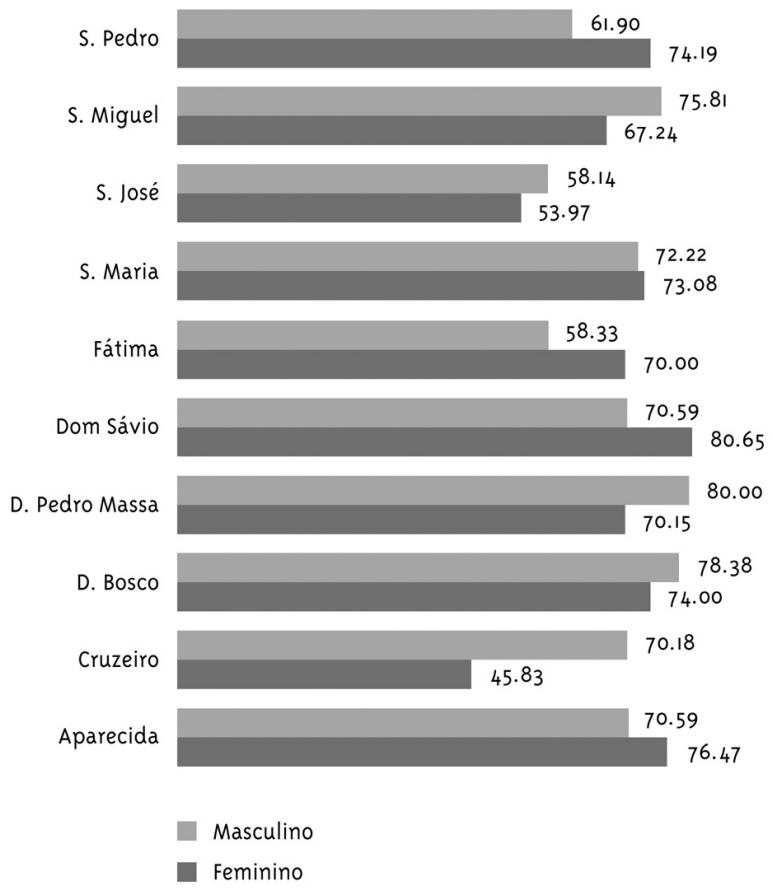

Figura 3 - Prevalência do número de parasitas por amostras de fezes, 2005-2006

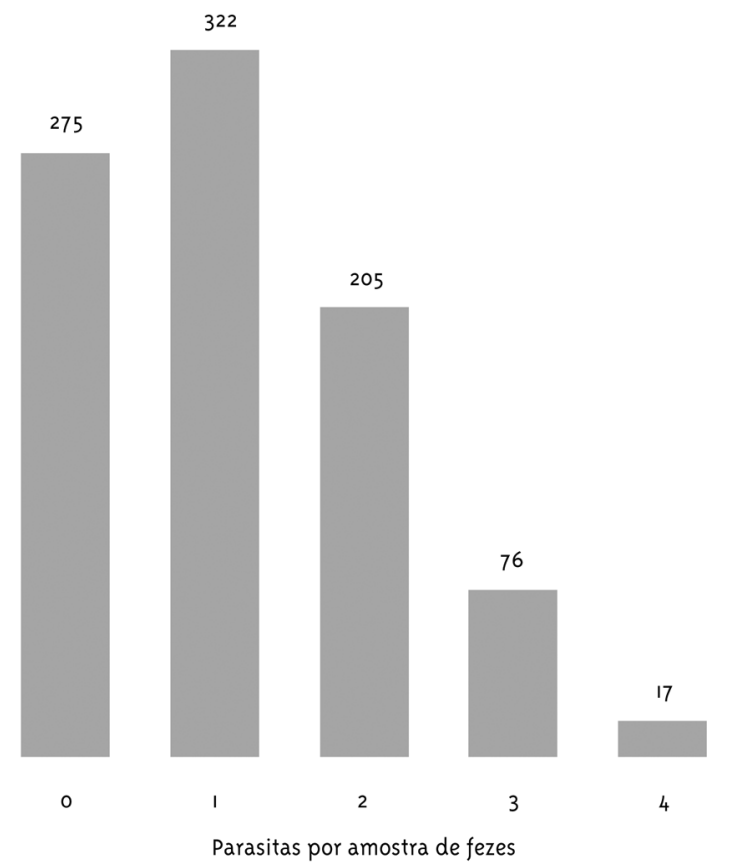

sitismo (duas ou mais espécies) em aproximadamente $33 \%(298 / 895)$ das amostras.

Na Tabela 3 estão listados os parasitos, suas prevalências específicas e suas proporções em relação ao total de sujeitos infectados (620). Portanto, 69,27\% dos indivíduos estavam infectados por algum parasito/comensal no momento da coleta dos dados. A. lumbricoides foi encontrado em aproximadamente $45 \%$ dos sujeitos. As espécies mais prevalentes foram, em ordem decrescente, A. lumbricoides, Entamoeba coli, Endolimax nana e Blastoyistis hominis.

\section{Tabela 3 - Prevalência total de parasitos em população amostrada da sede do distrito de lauaretê (AM), 2005-2006}

\begin{tabular}{|l|c|c|c|}
\hline Espécie & $n$ & $\begin{array}{c}\text { \% do total } \\
\text { de sujeitos }\end{array}$ & $\begin{array}{c}\text { \% do total } \\
\text { de amostras } \\
\text { positivas }\end{array}$ \\
\hline Ascaris lumbricoides & 402 & 44,92 & 64,84 \\
\hline Entamoeba coli & 202 & 22,57 & 32,58 \\
\hline Endolimax nana & 92 & 10,28 & 14,84 \\
\hline Blastocystis hominis & 83 & 9,27 & 13,39 \\
\hline E. histolitica / E. dispar & 66 & 7,37 & 10,65 \\
\hline Giardia lamblia & 53 & 5,92 & 8,55 \\
\hline Hymenolepis nana & 33 & 3,69 & 5,32 \\
\hline Ancilostoma duodenale & 31 & 3,46 & 5,00 \\
\hline Trichuris trichiura & 29 & 3,24 & 4,68 \\
\hline Iodamoeba butschlii & 16 & 1,79 & 2,58 \\
\hline Strongyloides stercoralis & 8 & 0,89 & 1,29 \\
\hline Enterobius vermicularis & 6 & 0,67 & 0,97 \\
\hline Chilomastix mesnili & 5 & 0,56 & 0,22 \\
\hline Entamoeba hartmanni & 2 & 61,32 \\
\hline
\end{tabular}

No total de 225 amostras de solo coletadas, cerca de $5 \%$ apresentaram resultado positivo na presença das 3 formas evolutivas de parasitos (cistos, larvas e ovos), $57 \%$ apresentaram resultado para a presença de alguma das formas parasitárias e 38\% apresentaram resultado negativo. Verificou-se que, dentre as amostras que apresentaram cistos, a proporção de $\varepsilon n t a-$ moeba coli foi de 52,6\% e de Giardia sp de 42,10\%; dentre as amostras de solo que tinham larvas, a proporção de ancilostomídeos foi de 52,6\%, enquanto dentre as que apresentaram ovos, as maiores proporções foram de Toxocara sp (58\%), Ascaris sp (52,6\%), Hymenolepis sp (47,36\%), Taenia sp (47,36\%) e Trichuris sp (47,36\%). 
Com relação à presença de ancilostomídeos no solo, no total de 10 vilas foram detectadas formas larvárias em $73 \%$ e ovos em $55 \%$ das vilas. Embora tenha sido diagnosticada uma gama de organismos no solo, como os cistos de protozoários Balantidium coli, $\varepsilon$. coli, $\varepsilon$. histolytica / E. dispar; Giardia sp, Iodamoeba sp, Isospora sp; as larvas de helmintos Ancylostoma sp, Enterobius sp, Strongyloides sp, Trichuris sp; e os ovos ou larvas de helmintos ancilostomideo(s), Ascaris sp, Echinococcus sp, Enterobius sp, Hymenolepis sp, Schistosoma sp, Taenia sp, Toxocara sp, Trichuris sp, na associação das formas de parasitos encontrados no solo com os indivíduos infectados, dos quatro parasitos de maior prevalência (Tabela 3), apenas $A$. lumbricoides e $\varepsilon$. coli detectados em amostras de solo por vila apresentaram correlação com indivíduos parasitados.

Na Tabela 1 sintetizaram-se os resultados referentes à A. lumbricoides em amostras fecais e em amostras no solo. Em relação à proporção desse parasito em amostras fecais positivas, os valores entre as vilas variaram entre $41 \%$ a $75 \%$ e as diferenças foram significativas $\left(X_{9}{ }_{9}=30,300 ; \mathrm{p}=0,0004\right)$. As proporções de amostras de solo contaminadas com A. lumbricoides foram inferiores às proporções desse parasito em amostras fecais. Foram significativas as diferenças de proporções de amostras de solo contaminadas com $A$. lumbricoides $\left(X_{9}{ }_{9}=21,467 ; \mathrm{p}=0,0107\right)$. Nas vilas Dom Bosco e São Miguel as diferenças entre proporções de amostras de solo contaminadas não foram significativas $\left(X_{1}^{2}=2,082 ; p=0,1491\right.$ e $X_{1}^{2}=1,902 ; p=0,1679$, respectivamente), ao passo que nas demais vilas houve diferenças significativas.

Os dados percentuais de prevalência de A. lumbricoides da Tabela 1, exibidos na Figura 4, mostraram uma associação positiva, sendo o coeficiente de correlação igual a o,476, porém esse valor não foi significativo ( $\mathrm{p}=\mathrm{o}, 1647)$.

Os resultados referentes à $\varepsilon$. coli em amostras fecais demonstraram que, em relação à prevalência, os valores entre as vilas variaram entre $68 \%$ a $88 \%$, superiores aos resultados de $A$. lumbricoides e as diferenças foram significativas $\left(X^{2}{ }_{9}=19,169 ; p=0,0238\right)$. As proporções de amostras de solo contaminadas foram inferiores às proporções de $\varepsilon$. coli em amostras fecais. Ao contrário do que se apurou com A. lumbricoides, as diferenças de proporções de amostras de solo

\section{Figura 4 - Prevalência de sujeitos versus prevalência de amostras de solo com Ascaris lumbricoides de acordo com a vila de procedência, 2005-2006}

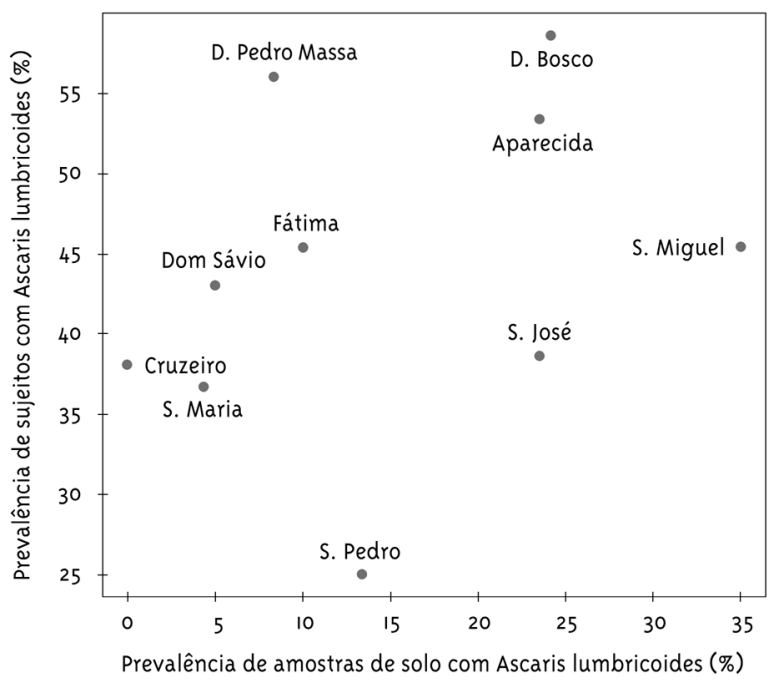

contaminadas com $\varepsilon$. coli nas diferentes vilas não foram significativas $\left(X^{2}{ }_{9}=9,949 ; \mathrm{p}=0,3547\right)$. Em todas as vilas houve diferenças significativas entre a prevalência em amostras fecais e as prevalências em amostras de solo contaminadas ( $\mathrm{p}<0,00005$ em todas as comparações).

\section{Discussão}

Do ponto de vista de saúde pública, verificou-se que a população indígena do distrito de Iauaretê encontrava-se exposta aos riscos de infecção, em decorrência da falta de saneamento básico, da aglomeração populacional local e da manutenção de alguns hábitos sanitários tradicionais, como a disposição de dejetos humanos e de resíduos sólidos em áreas peri-domiciliares ou próximas a fontes de água de consumo humano. Verificou-se também a existência de uma pequena infra-estrutura urbana local como posto de saúde, escola, agência dos correios, fornecimento de energia elétrica a base de gerador, canais de televisão, um pequeno comércio, um Pelotão de Fronteira do Exército Brasileiro e uma Missão Salesiana da Igreja Católica.

Por meio de diagnóstico ambiental, pode-se identificar a falta de tratamento de água domiciliar, sendo esta proveniente de poços rasos ou nascentes, chuva, biqueira de poço profundo, igarapé e rio. Como re- 
latado anteriormente, a população indígena utilizava para as necessidades fisiológicas áreas peri- domiciliares, roças, igarapés e rios. Para banho, os indígenas possuíam o hábito de utilizar o próprio rio Waupés e igarapés. Quanto aos resíduos sólidos, encontravamse, na maioria das vezes, dispersos no ambiente de forma irregular, como próximos de fontes de captação de água ou sobre o solo, pois não havia um sistema de coleta de resíduos. Os moradores se viam obrigados a dar uma destinação precária ao material gerado, que na maioria das vezes incluía a queima, o enterramento, o acúmulo sobre o solo e o despejo no rio ou nos igarapés (Giatti e col., 2006). Portanto, a disposição de dejetos humanos e de resíduos sólidos em áreas peri-domiciliares e próximas às nascentes, o consumo de água sem tratamento, o grau de contaminação das águas por coliformes termotolerantes (89,2\%), associados à alta prevalência de parasitos intestinais, ao crescente processo de urbanização e à falta de saneamento têm afetado negativamente a qualidade de vida da população indígena de Iauaretê.

No que se refere à elevada prevalência de parasitos intestinais $(69 \%)$ dentre a população estudada, Bóia e col. (1999) também verificaram a prevalência de 87,6\% em Novo Airão (AM). Em Coari, no Rio Solimões, Coura e col. $(1993,1994)$ verificaram índice de $68,9 \%$ e em Barcelos, no Rio Negro, 69,4\%, demonstrando que as populações indígenas de maneira geral sofrem com o enteroparasitismo, o que confirma as precárias condições de saúde pública dessas populações. Esse problema é apontado por Buchillet (2004), em que dados epidemiológicos disponíveis sobre a região do Alto Rio Negro, como um todo, indicam predomínio de doenças infectocontagiosas e parasitárias, com destaque para afecções do aparelho respiratório e digestivo, malária, dermoparasitoses e piodermites.

Para Miranda e col. (1998) as espécies A. lumbricoides e $\varepsilon$. histolytica $/ \mathcal{E}$. dispar foram os helmintos e os protozoários de maior prevalência. Estudos tanto em comunidades indígenas como em não-indígenas têm apresentado diversos resultados, com variação de prevalência entre $A$. lumbricoides e ancilostomídeos, alertando para o elevado risco de formas invasivas intestinais e extra-intestinais de amebíase (Andrade e Andrade Júnior, 1996; Cardoso e col., 1995; Coimbra e Mello, 1981; Collins e Edwards, 1981; Gonçalves e col., 1990; Kobayashi e col., 1995).
De acordo com Bóia e col. (1999) as parasitoses intestinais transmitidas por água e alimentos como o A. lumbricoides (35,1\%), E. histolytica/E. dispar (29,1\%) e G. lamblia (17,4\%) apresentam altos índices de prevalência, e as transmitidas por penetração de larvas na pele, como ancilostomídeos (4,7\%) e Strongyloides stercoralis (2,5\%) apresentam menor prevalência.

No distrito de Iauaretê a maior prevalência foi também de $A$. lumbricoides (44,9\%) seguido por $\varepsilon$. coli (22,6\%), E. nana (10,3\%) e B. hominis (9,3\%). As menores incidências foram de $S$. stercoralis (o,89\%), $\varepsilon$. vermicularis (o,67\%) e Chilomastix mesnili (o,56\%).

A contaminação de solo e parasitose por A. lumbricoides apresentaram correlação positiva $(0,476)$, porém não significativa $(\mathrm{p}=0,1647)$. No caso dos geohelmintos presentes nas amostras de solo como Ascaris e ancilostomídeos, são requisitos ecológicos alguns fatores como o microclima do solo, a presença em abundância de formas infectantes no solo e os indivíduos susceptíveis ao parasitismo, persistindo infecções e garantindo o ciclo de transmissão de parasitos intestinais em Iauaretê. (Whitfield, 1993; Cutolo e col., 2006).

A presença de protozoários e helmintos no solo representa um risco à saúde humana em cada localidade. Apesar dos diferentes ciclos biológicos de cada espécie de enteroparasito, as condições ambientais como temperaturas entre 25 e $30^{\circ} \mathrm{C}$ e umidade elevada propiciam o desenvolvimento e a manutenção dos seus estágios evolutivos. Alia-se a ausência de saneamento e de práticas de higiene, que favorecem a dispersão desses agentes no ambiente, assegurando a continuidade da relação parasito-hospedeiro (WHO, 1991; Heller, 2000; Cutolo e col., 2006).

Segundo Ruppel e Doenhoff (1998), a interação parasito-hospedeiro pode envolver estimulação e evasão dos mecanismos de defesa do hospedeiro. No caso dos parasitos encontrados no solo, a presença é considerada um risco potencial em detrimento da baixa imunidade da população, insalubridade no ambiente urbano e a inadequada disposição de resíduos sólidos, ocorrência de córregos contaminados, dentre outros eventos ambientais que ameaçam, prejudicam ou desafiam o bem-estar do organismo (Elliott e col., 1993).

Neste estudo foi verificado que os indivíduos têm a mesma prevalência independentemente de sexo e idade. Miranda e col (1998) verificaram também não 
haver correlação entre sexo e parasitismo e idade e parasitismo em uma aldeia indígena Parakanã no sudeste do Pará, apesar das diferenças de atividade entre os sexos. Porém, houve diferença significativa quando comparadas à vila Aparecida e às vilas Cruzeiro e São José, indicando que os seus habitantes têm menores chances de estar infectados do que os da vila Aparecida.

Chernella e Thatcher (1989) verificaram, por meio de inquérito parasitológico intestinal dentre indígenas da região do Rio Waupés, que populações sedentárias, de hábitos ribeirinhos, apresentaram piores indicadores de saúde, sob aspectos de parasitos intestinais, do que populações que ainda conservavam hábitos seminômades. No presente estudo, a única etnia de hábitos seminômades dentre a população estudada foi a Hupda, habitante da vila Fátima, onde, apesar de vivenciarem situações semelhantes quanto às práticas de higiene e saneamento, apresentaram os menores percentuais de prevalência de parasitos na sede do distrito.

Para Miranda e col. (1998) a preocupação maior é a grande proporção de indivíduos poliparasitados. Mais da metade da população, em todas as idades e em ambos os sexos, alberga duas ou mais espécies de parasitos na aldeia Parakanã. Em Iauaretê cerca de $36 \%$ da população amostrada teve prevalência de apenas um parasito e em aproximadamente $33 \%$ foram encontrados dois ou mais parasitos

No distrito de Iauaretê, segundo dados de morbidade obtidos no DSEI/Pólo Base de Iauaretê, no período de maio a dezembro de 2003 , as doenças diarréicas e as parasitoses intestinais representaram cerca de $25 \%$ do total dos casos de moléstias notificados.

A partir de levantamento em registros de internações hospitalares da população indígena atendida no DSEI Porto Velho (RO), Escobar e col. (2003) afirmam que as doenças infecciosas e parasitárias foram a segunda causa mais freqüente de internação $(15,6 \%$ do total de internações), dentre esse grupo, $41 \%$ dos acometimentos foram diarréias, moléstias que desempenham importante papel no perfil de morbi-mortalidade de populações indígenas de Rondônia, sobretudo entre crianças de 0-5 anos de idade.

Quanto aos reflexos dessas situações em indicadores de saúde, Gióia (1995) identificou fragilidades quanto ao saneamento básico e ao conhecimento da população sobre a transmissão de verminoses, associando esses aspectos negativos às elevadas prevalências de parasitos e comensais intestinais na ordem de 73,5\% entre a população investigada em área de mata Atlântica, demonstrando o poliparasitismo com média de 2,3 parasitos por indivíduo.

\section{Considerações Finais}

Os altos índices de prevalência de parasitos intestinais, a grande quantidade de indivíduos com poliparasitismo, a qualidade da água utilizada pela população, as precárias condições de saneamento, ou a sua falta, em muitos casos, e determinados hábitos inapropriados para a atual situação de concentração populacional demonstram os riscos reais na saúde das populações indígenas e a necessidade de medidas urgentes de saneamento ambiental. Esse fato é também mencionado por Buchillet (2004), que observou características socioculturais como concentração populacional, pouca mobilidade espacial e hábitos de higiene, e precárias condições de saneamento em que se encontram a maioria das comunidades indígenas em decorrência do processo de contato com os não-índios. Nos grupos de indígenas com elevado grau de mobilidade espacial, as condições sanitárias são melhores que aquelas dos grupos sedentários. Em Iauaretê essa relação se faz com a população da vila Fátima, de etnia Hupda, que tradicionalmente mantém maior mobilidade e menor concentração populacional.

Diante deste estudo, no caso de áreas indígenas, a implantação de sistemas de saneamento ambiental deve considerar as características geográficas locais, as reais necessidades da população e, principalmente, a cultura e os hábitos desses povos. Além disso, recomenda-se o desenvolvimento de processos de educação em saúde baseados no constante diálogo entre o saber tradicional e o saber científico norteador da problemática e das soluções, visando romper ciclos transmissores de doenças e a melhoria das condições de vida da população local como um todo.

\section{Agradecimentos}

Os autores agradecem à Fundação Nacional de Saúde (Funasa) pelo financiamento do projeto; ao Distrito Sanitário Especial Indígena (DSEI/ARN/FOIRN); ao 
Luciano M. Giatti pelo trabalho voluntário de coleta de amostras de solo em campo, e ao VII Comando Aéreo Regional da Força Aérea Brasileira (VII Comaer/ FAB) pelo apoio logístico e de transporte.

\section{Referências}

ASSOCIAÇÃO BRASILEIRA DE NORMAS TÉCNICAS

(ABNT). NBR 10oo7: solo e resíduos sólidos: amostragem de resíduos: procedimento. Rio de Janeiro, 1987.

ANDRADE, D. R.; ANDRADE JÚNIOR, D. R. Amebíase. In: VERONESI, R.; FOCCACIA, R. (Org.). Tratado de infectologia. São Paulo: Atheneu, 1996. p. 1149-1159.

ANDRELLO, G. L. Iauaretê: transformações sociais e cotidiano no Rio Waupés, Alto Rio Negro, Amazonas. 2004. Tese - Instituto de Filosofia e Ciências Humanas da Universidade de Campinas, Campinas, 2004 .

ANDRELLO, G. L.; BUCHILLET, D.;AZEVEDO, M. (Coord.). Levantamento sócioeconômico, demográfico e sanitário de Iauaretê/Centro: primeiro relatório.

São Gabriel da Cachoeira: ISA, 2002.

AMERICAN PUBLIC HEALTH ASSOCIATION (APHA). Standard methods for the examination of water and wastewater. 20. ed. New York, 1999.

BÓIA, M. N. et al. Estudo das parasitoses intestinais e da infecção chagásica no município de Novo Airão, estado do Amazonas, Brasil. Cadernos de Saúde

Pública, Rio de Janeiro, v. 15, n. 3, p. 497-504, 1999.

BUCHILLET, D. Saúde \& povos indígenas. Cadernos de Saúde Pública, Rio de Janeiro, v. 11, n. 2, p. 338 341, 1995.

BUCHILLET, D. Cultura e saúde pública: reflexões sobre o Distrito Sanitário Especial Indígena do Rio Negro. In: LANGDON, E. J.; GARNELO, L. (Org.). Saúde dos povos indígenas: reflexões sobre antropologia participativa. Rio de Janeiro: Associação Brasileira de Antropologia, 2004. p. 53-67.

CARDOSO, G. S.; SANTANA, A. D. C.; AGUIAR, C. P. Prevalência e aspectos epidemiológicos da giardíase em creches no município de Aracaju, SE, Brasil. Revista de Sociedade Brasileira de Medicina Tropical, Brasília, DF, v. 28, p. 25-31, 1995.
CHERNELA, J. M.; THATCHER, V. E. Comparison of parasite burdens in two native amazonian populations. Medical Anthropology, New York, v. 10, p. 279-285, 1989.

COIMBRA, C. E. A.; MELLO, D. A. Enteroparasitas e Capillaria sp. entre o grupo Suruí, Parque Indígena Aripuanã, Rondônia. Memórias do Instituto Oswaldo Cruz, Rio de Janeiro, v. 76, p. 299-302, 1981.

COLLINS, R. F.; EDWARDS, L. D. Prevalence of intestinal helminths and protozoans in a rural population segment of the Dominican Republic. Transactions of the Royal Society of Tropical Medicine and Hygiene, London, v. 75, p. 549-551, 1981.

COSTA, D. C. Política indigenista e assistência à saúde: Noel Nutels e o Serviço de Unidades Sanitárias Aéreas. Cadernos de Saúde Pública, Rio de Janeiro, v. 4, n. 3, p. 388-401, 1987.

COURA, J. R et al. Aspectos epidemiológicos, sociais e sanitários em áreas do Médio Solimões: II - estudo de dois bairros periféricos da cidade de Coari e quatro localidades do lago do Mamiá, estado do Amazonas. Anais da Academia Nacional de Medicina, Rio de Janeiro, v. 153, p. 183-186, 1993.

COURA, J. R. et al. Aspectos epidemiológicos, sociais e sanitários de uma área no Rio Negro, estado do Amazonas, com especial referência às parasitoses intestinais e à infecção chagásica. Cadernos de Saúde Pública, Rio de Janeiro, v. 10, p. 327-336, 1994. CUTOLO, S. A.; MATTÉ, M. H.; ROCHA, A. A. Monitoring of parasitological contamination in treated wastewater from activated sludge system. Management of Environmental Quality, Bradford, v. 17, n. 1, p. 43-56, 2006.

ELLIOTT, S. J.; TAYLOR, S. M.; WALTER, S. Modeling psychosocial effects of exposure to solid waste facilities. Social Science and Medicine, Amsterdam, v. 37, n. 6, p. 790-804, 1993.

ESCOBAR, A. L. et al. Causas de internação hospitalar indígena em Rondônia: o distrito sanitário especial indígena Porto Velho. In: COIMBRA JR, C. E. A.; SANTOS, R. V.; ESCOBAR, A. L. Epidemiologia e saúde dos povos indígenas no Brasil. Rio de Janeiro: Fiocruz: Abrasco, 2003. p. 127-147. 
FEDERAÇÃO DAS ORGANIZAÇÕES INDÍGENAS DO RIO NEGRO/INSTITUTO SOCIOAMBIENTAL (FOIRN/ISA). Povos indígenas do alto e médio Rio Negro: uma introdução à diversidade cultural e ambiental do noroeste da Amazônia brasileira. 2. ed. São Gabriel da Cachoeira, 2000.

GIATTI, L. L. et al. Condições sanitárias e socioambientais em Iauaretê, área indígena em São Gabriel da Cachoeira/AM. Ciência \& Saúde Coletiva, Rio de Janeiro, 2006. Disponível em: <http:// www.abrasco.org.br/cienciaesaudecoletiva/artigos/ artigo_int.php?id_artigo=438>. Acesso em: 12 nov 2006.

GIOIA, I. Levantamento eco-parasitológico da população residente na Fazenda Intervales, SP. 1995. Tese - Faculdade de Saúde Pública da Universidade de São Paulo, São Paulo, 1995.

GONÇALVES, J. F. et al. Parasitological and serological studies on amoebiasis and other intestinal parasitic infections in the rural sector around Recife, Northeast Brazil. Revista do Instituto de Medicina Tropical de São Paulo, São Paulo, v. 32, p. 428-435, 1990.

HELLER, L. Saneamento e saúde. Brasília, DF: OPAS/ OMS, 2000.

KOBAYASHI, J. et al. Prevalence of intestinal parasitic infection in five farms in Holambra, São Paulo, Brazil. Revista do Instituto de Medicina Tropical de São Paulo, São Paulo, v. 37, p. 13-18, 1995.

LEVENTHAL, R.; CHEADLE, R. F. Medical parasitology: a self-instructional text. 4. ed. Philadelphia: F. A. Davis, 2000.
LUTZ, A. O Schistosomum mansoni e a schistosomatose segundo observações feitas no Brasil. Memórias do Instituto Oswaldo Cruz, Rio de Janeiro, v. 11, p. 121-155, 1919.

MIRANDA, R. A.; XAVIER, F. B.; MENEZES, R. C. Parasitismo intestinal em uma aldeia indígena Parakanã, sudeste do estado do Pará, Brasil.

Cadernos de Saúde Pública, Rio de Janeiro, v. 14, n. 3 , p. 507-511, 1998.

ORGANIZACIÓN MUNDIAL DE LA SALUD (OMS). Métodos básicos de laboratorio en parasitologia medica. Genebra, 1992.

PETERS, W.; GILLES, H. M. Color atlas of tropical medicine and parasitology. 4. ed. New York: Mosby Wolfe, 1995.

R DEVELOPMENT CORE TEAM. A language and environment for statistical computing. Vienna: R Foundation for Statistical Computing, 2005.

RUPPEL, A.; DOENHOFF, M. J. Vector biology and the control of parasitic diseases. Parasitology Today, Amsterdam, v. 14, n. 8, p. 299-300, 1998.

SANTOS, R. V.; COIMBRA JR, C. E. A. Cenários e tendências da saúde e da epidemiologia dos povos indígenas no Brasil. In: COIMBRA JR, C. E. A.; SANTOS, R. V.; ESCOBAR, A. L. (Org.). Epidemiologia e saúde dos povos indígenas no Brasil. Rio de Janeiro: Fiocruz: Abrasco, 2003. p. 13-47.

WHITFIELD, P. I. Parasitic helminths. In: COX, F. E. G. Modern parasitology: a textbook of parasitology. 2. ed. Oxfor: Blackwell Science, 1993. p. 24-52.

WORLD HEALTH ORGANIZATION. Environmental health in urban development. Geneva, 1991.

(Technical Report Series, 8o7). 